\title{
Du retrait à la reconquête : pratiquer la ville après un épisode psychotique
}

\author{
Zoé Codeluppi \\ Institute of Geography, University of Neuchâtel, Neuchâtel, 2000, Switzerland \\ Correspondence: Zoé Codeluppi (zoe.codeluppi@unine.ch)
}

Received: 14 January 2018 - Revised: 13 January 2019 - Accepted: 17 January 2019 - Published: 13 February 2019

\begin{abstract}
Résumé. The article aims to provide a better understanding of the urban practices of young people living with a diagnosis of psychosis while recovering. I show the way practices are adjusted according to the temporal dynamics of psychosis. I argue that the continuous variability of symptoms over the recovery period implies alternately practices of withdrawal and reconquest of the urban space. I first outline participants' reconquest of urban spaces, which starts in well-known places and then extends to less familiar ones. In doing so, I point out the diversity of urban spaces inhabited by participants during the recovery process which includes institutional, private, as well as public places. I then outline the various material, relational and sensory resources available in these spaces. I show how participants use them according to the temporal dynamics. I finally highlight the way participants are gradually getting involved in the relationship with a large array of resources as the intensity of symptoms is reducing. My analysis is based on a three months ethnography in a therapeutic institution in Lausanne.
\end{abstract}

\section{Introduction}

«J'aime bien venir dans ce parc... ça me calme. Je passe souvent à travers le parc ... avant d'aller à l'institution. C'est un endroit à part, un peu isolé ça me permet de réfléchir. Quand ça va pas bien... je viens ici... je m'assieds sur un banc à côté de la fontaine ou de l'autre côté vers la cascade ... je fais abstraction de tout le reste ... ça m'aide d'être ici » (Nathan, Extrait du cahier des observations, juin 2016).

Nathan ${ }^{1}$ est un jeune homme vivant avec un diagnostic de schizophrénie. Il se rend depuis une année, chaque jour, dans une institution thérapeutique au centre de Lausanne. Nathan pratique quotidiennement la ville dans le cadre de ses déplacements et de ses activités. Mais tout comme les autres jeunes gens fréquentant l'institution, ce jeune homme de vingt-deux ans ne pratique pas l'espace urbain de façon aléatoire. Le vécu des troubles psychotiques influe en effet sur les façons de mobiliser l'environnement. Nathan m'explique que lorsque les troubles se manifestent, il préfère rester en retrait, loin des autres et du bruit. Dans ces moments-là, le

\footnotetext{
${ }^{1}$ En raison du caractère sensible des données, les noms des jeunes gens et des soignants ont été anonymisés.
}

jeune homme privilégie des espaces calmes et apaisants qui l'aident à se ressourcer. Cet acte de retrait n'est pas propre à Nathan, il témoigne plus largement des pratiques caractéristiques des jeunes personnes souffrant de schizophrénie.

Dans cette perspective, l'article vise à mettre en évidence les pratiques de retrait et de reconquête urbaine de jeunes personnes souffrant de troubles psychotiques et plus précisément de schizophrénie ${ }^{2}$, au cours de leur rétablissement. Les pratiques désignent les différentes formes de mobilisation de l'environnement, conscientes et inconscientes, qui sont développées de façon récurrente par les jeunes gens vivant avec un diagnostic de schizophrénie dans leur expérience de la ville (Söderström et al., 2017). Le rétablissement fait quant à lui référence au lent processus d'autonomisation des personnes souffrant de schizophrénie. Celui-ci se caractérise par une stabilisation ou par l'atténuation progressive de l'inten-

\footnotetext{
${ }^{2}$ La psychose est un trouble mental qui se caractérise par une perte de contact avec la réalité. Il existe différents troubles psychotiques, dont la schizophrénie ou le trouble bipolaire par exemple. Dans le cadre de cette étude, j'ai inclus l'ensemble des jeunes gens souffrant de schizophrénie. Ce trouble se traduit par des distorsions de la pensée, des perceptions, des émotions, du langage et du comportement.
} 
sité et de la fréquence des troubles, menant parfois à une disparition totale des symptômes ${ }^{3}$ (Bister et al., 2016; Klausner, 2014).

Dans le cadre de ce travail, je mets dans un premier temps en évidence la temporalité non-linéaire et fluctuante du rétablissement des troubles de la schizophrénie qui est à l'origine des pratiques de retrait et de reconquête urbaine. La temporalité du rétablissement désigne le laps de temps s'étendant depuis la période de crise psychotique à celle de stabilisation ou de rémission des troubles (Klausner, 2014). À cet effet, je montre l'existence d'une temporalité courte et d'une temporalité longue du rétablissement. La temporalité courte se caractérise par de brèves périodes de manifestation des troubles qui interviennent de façon récurrente au cours du processus long de rémission ou de stabilisation de symptômes. La temporalité longue fait référence au long processus de rémission progressive des symptômes. Mon analyse démontre que les pratiques de retrait, caractéristiques des périodes de manifestation des troubles, évoluent progressivement vers des pratiques de reconquête urbaine, au fur et à mesure du long processus d'atténuation des symptômes.

Dans un deuxième temps, je montre que l'évolution des pratiques témoigne, d'une part, de l'extension du périmètre de pratiques des jeunes schizophrènes et se traduit, d'autre part, par la mobilisation d'une diversité de ressources de l'environnement. Je souligne à cet effet que la spatialité du rétablissement, c'est-à-dire le rapport entre les pratiques des individus et les diverses «prises» offertes par un environnement donné (Golembiewski, 2013), s'élargit progressivement vers de nouveaux espaces urbains aux ressources multiples et variées ${ }^{4}$. Les «prises» renvoient à des ressources matérielles, sociales et sensorielles mobilisées par les jeunes gens au cours de leur rétablissement (Golembiewski, 2013). Dans cette perspective, la spatialité du rétablissement est à la fois façonnée à travers les pratiques des individus et déterminée par la réalité matérielle de l'espace. Celui-ci est donc à la fois produit et vécu par les individus. Ces derniers ont une capacité à agir sur leur environnement mais ils sont également contraints par les caractéristiques intrinsèques à l'environnement (Lefèbvre, 1974).

Dans un troisième temps, je mets en évidence que les pratiques de retrait et d'engagement révèlent un degré d'engagement particulier des jeunes gens avec leur environnement. Mon analyse montre qu'au fur et à mesure de la stabilisation

\footnotetext{
${ }^{3}$ La stabilisation des troubles ou des symptômes désigne un état stable à durée variable, au cours duquel les symptômes sont toujours présents mais moins intenses. Chez certaines personnes les symptômes disparaissent progressivement au fil du temps, alors que chez d'autres ils réapparaissent à nouveau avec une intensité généralement plus forte.

${ }^{4}$ Golembiewski emploie le terme de «positive affordance » pour désigner les ressources de l'environnement (Golembiewski, 2013). J'ai choisi de traduire ce terme par «prise» permettant de souligner la dimension à la fois produite et vécue de l'espace urbain par des acteurs.
}

des troubles, les individus accroissent et diversifient les ressources mobilisées et témoignent ainsi d'une intensification de leur degré d'engagement avec l'environnement. Afin de rendre compte de manière fine du degré d'investissement au cours des pratiques de retrait et de reconquête du rétablissement, je mobilise le concept «d'intensité d'engagement» développé par le sociologue Laurent Thévenot (2006). Ce concept désigne les différentes façons de s'investir dans une relation avec l'environnement et de se coordonner avec celuici en fonction de la portée de l'action. Plus celle-ci est collective, plus elle requiert un investissement intense et un degré de coordination élevé de l'individu avec son environnement. À l'aide du concept de Thévenot, il s'agit donc de montrer comment, au cours de leur rétablissement, les jeunes personnes souffrant de schizophrénie s'impliquent progressivement dans des pratiques plus collectives qui requièrent un degré d'engagement plus marqué avec l'environnement.

Cet article cherche à élargir le champ de recherche en géographie, en proposant une réflexion sur les pratiques urbaines de jeunes personnes souffrant de schizophrénie après un premier épisode psychotique ${ }^{5}$, dans un contexte suisse qui fait pour l'instant l'objet de peu d'études. Les travaux actuels en géographie de la santé mentale s'attachent en effet essentiellement à décrire les pratiques de jeunes personnes psychotiques dans des contextes américains, australiens, néo-zélandais, allemands et britanniques (Parr, 2006, 2008 ; Philo et Wolch, 2001 ; Knowles, 2000 ; Estroff, 1985 ; Gleeson, 1999). De même, les temporalités dans lesquelles s'ancrent ces pratiques ne sont que partiellement analysées, bien qu'elles influencent de façon déterminante les trajectoires spatiales de rétablissement des jeunes personnes souffrant de schizophrénie. Dans le but d'enrichir la réflexion sur les temporalités et les spatialités de rétablissement, l'article mobilise une littérature plus large en sciences sociales, qui traite des dimensions temporelles et spatiales des personnes souffrant de schizophrénie (Duff, 2012; Klausner, 2014 ; Corin, 2003 ; Bister et al., 2016).

Toutefois, ces travaux défendent majoritairement une vision linéaire de la temporalité des troubles, allant du vécu d'un épisode psychotique à la période de stabilisation des troubles (Evered, 2016; Poirel et al., 2013). Même si une partie de ces analyses reconnaît l'existence de variations temporelles au cours du rétablissement, celles-ci ne sont pas documentées de façon précise et détaillée (Yates et al., 2012).

Par ailleurs, les analyses en sciences sociales portant sur la spatialité du rétablissement et en particulier sur les espaces dans lesquels s'inscrivent les pratiques de retrait et de reconquête sont partielles. Ces recherches traitent essentiellement des espaces institutionnels de soins, c'est-à-dire de l'ensemble des lieux de soins formels et thérapeutiques fréquentés pendant ou juste après l'hospitalisation (Poirel et al., 2013). En revanche, la fonction de l'espace urbain du quo-

\footnotetext{
${ }^{5}$ L'épisode psychotique se définit comme la manifestation la plus aiguë des troubles.
} 
tidien, hors institution, dans le processus de rétablissement n'est pas traitée par cette littérature (Evered, 2016). Mon analyse propose d'explorer le rôle de l'espace urbain du quotidien et en particulier des espaces publics dans le processus de rétablissement des jeunes personnes psychotiques (Evered, 2016; Klausner, 2014 ; Bister et al., 2016).

L'article s'organise en trois parties. Dans une première section, je situe la recherche par rapport à la littérature existante sur les pratiques, les espaces et les temporalités de rétablissement de personnes vivant avec un diagnostic de schizophrénie en géographie et en sciences sociales. Puis, sur la base de mes données ethnographiques, je mets en évidence, dans un deuxième temps, l'évolution des spatialités et des pratiques de rétablissement des jeunes personnes souffrant de schizophrénie en fonction de la temporalité courte et longue du rétablissement. J'élabore à cet effet une typologie qui permet de rendre compte des ressources mobilisées dans les pratiques quotidiennes des jeunes gens en fonction des deux temporalités du rétablissement, renseignant par extension l'intensité d'engagement avec leur environnement.

En guise de conclusion, je souligne le potentiel thérapeutique de l'espace public urbain et je propose quelques pistes de réflexion destinées aux professionnels de la santé mentale pour le développement de nouvelles stratégies thérapeutiques en ville. Mon étude se base sur une ethnographie menée dans une institution thérapeutique avec des jeunes personnes ayant vécu un épisode psychotique remontant à moins de trois ans.

\section{Schizophrénie et milieu urbain}

Pour mieux saisir l'intérêt et la pertinence de mon analyse des pratiques urbaines, il faut d'abord examiner la relation des personnes souffrant de troubles psychotiques avec le milieu urbain ${ }^{6}$. Ce dernier joue en effet un rôle dans le développement de psychoses. La première étude montrant cette relation est menée en 1939 par Faris et Dunham. Celle-ci met en évidence la concentration plus élevée de personnes souffrant de schizophrénie dans le centre de la ville de Chicago par à rapport d'autres zones de l'agglomération (Faris et Dunham, 1939). Par la suite, d'autres études plus récentes en psychiatrie montrent que le risque de développer des troubles psychotiques est plus élevé en ville qu'en milieu rural (van Os, 2004 ; Kirkbride et al., 2007; Kelly et al., 2010; Vassos et al., 2012). Ce phénomène reste vérifié après contrôle des variables potentiellement explicatives, telles que la consommation de cannabis, le statut social et économique ou encore l'appartenance à une minorité ethnique (Kelly et al., 2010; Vassos et al., 2012). Cependant, la nature des mécanismes impliqués dans cette relation reste peu claire (Söderström et al., 2016).

D'autre part, plusieurs études montrent que les personnes souffrant de troubles psychotiques expérimentent souvent

\footnotetext{
${ }^{6}$ Dans le cadre de cette analyse, le milieu urbain fait référence aux zones du centre-ville à forte densité humaine et bâtie.
}

une intensification des symptômes en milieu urbain en raison de la forte sensibilité qu'elles développent aux stimulations de l'environnement (Kapur, 2003 ; Van Os et al., 2008). Les personnes vivant avec un diagnostic de schizophrénie sont en effet émotionnellement plus actives et plus sensibles que le reste de la population (Myin-Germeys et al., 2009). Dans cette perspective, les variations, même minimes, dans les interactions avec l'environnement influent sur l'intensité des expériences psychotiques. Les troubles ne se manifestent cependant pas de façon continue. Ils occupent environ un tiers du temps des individus (Myin-Germeys et al., 2009). Cela signifie que les troubles fluctuent constamment et de façon quotidienne, entraînant des changements dans la façon dont ces personnes s'engagent dans les activités sociales et spatiales (Myin-Germeys et al., 2009; Verdoux, H. et Cougnard, A., 2003).

\subsection{Analyses spatiales en géographie de la santé mentale}

De manière générale, il existe peu d'analyses en géographie de la santé mentale qui traitent des pratiques urbaines de jeunes personnes psychotiques. La plupart des études porte essentiellement sur les espaces fréquentés dans les phases aiguës de manifestation des troubles (Parr, 2006, 2008 ; Estroff, 1985 ; Knowles, 2000 ; Klausner, 2014). Ces recherches s'attachent à décrire les dimensions sociales et spatiales des espaces institutionnels de soins ${ }^{7}$ hérités de la désinstitutionalisation $^{8}$ (Parr, 2008 ; Milligan et Wiles, 2010). En revanche, le rôle des espaces urbains du quotidien dans les trajectoires de rétablissement des jeunes personnes vivant avec un diagnostic de schizophrénie est peu traité par cette littérature.

Par ailleurs, la littérature existante ne détaille pas le diagnostic des personnes étudiées. Cette absence d'indications précises ne permet ni de situer l'expérience psychotique des patients en fonction de l'apparition des troubles, ni de distinguer les situations chroniques des situations épisodiques. Ces informations sont pourtant indispensables à la compréhension des pratiques et des temporalités des jeunes personnes souffrant de troubles psychotiques.

\footnotetext{
${ }^{7}$ Les espaces institutionnels de soins désignent l'ensemble des établissements et des structures thérapeutiques de prise en charge psychiatrique. Parmi les espaces institutionnels, on trouve l'hôpital psychiatrique, les consultations ambulatoires et les hôpitaux de jour. Les différentes structures communautaires héritées de la désinstitutionalisation en font également partie, puisque la plupart de ces lieux font office d'hôpital de jour. Ces lieux peuvent être étatiques ou privés.

${ }^{8}$ Le processus de désinstitutionalisation se caractérise par la fermeture des grandes institutions asilaires au profit de petites structures de soins qui se basent sur le modèle ambulatoire, plus flexible, ouvert et proche des patients. Ce processus se développe d'abord de manière précoce dans les années 1930 aux États-Unis et dès les années 1950 en Europe.
} 
Dans le but de pallier l'absence de littérature sur les pratiques et sur les temporalités en géographie de la santé mentale, cet article mobilise une littérature interdisciplinaire plus large en sciences sociales. Celle-ci permet d'apporter et d' affiner la compréhension des spatialités, des temporalités et des pratiques des jeunes personnes souffrant de troubles psychotiques.

\subsection{Pratiques urbaines et temporalités de la psychose en sciences sociales}

Mon analyse s'appuie sur des recherches menées en sciences sociales (en ethnographie, en anthropologie, en psychologie sociale et en sociologie de la santé), qui traitent des pratiques urbaines et de la temporalité des troubles psychiques (Corin, 2002 ; Klausner, 2014; Evered, 2016; Poirel et al., 2013; Duff, 2015). En se basant sur l'expérience et le vécu des personnes vivant avec des troubles psychotiques, cette littérature montre la façon dont ces individus composent quotidiennement avec leur environnement matériel et social. L'environnement est considéré comme un vaste champ de ressources matérielles, sociales et sensorielles avec lesquelles les individus interagissent selon leurs besoins (Duff, 2012). Les ressources matérielles font référence à l'organisation d'un espace et à ses composantes physiques. Les ressources sociales désignent l'ensemble des potentiels relationnels permettant la création de contact offerts par un espace. Enfin, les ressources sensorielles regroupent l'ensemble des sollicitations auditives, visuelles et du toucher présentes dans l'environnement et qui participent au bien-être des individus (Duff, 2014). Ces trois types de ressources constituent de véritables «prises » que les personnes mobilisent en fonction de leurs besoins au cours de leur rétablissement (Golembiewski, 2013 ; Duff, 2014).

Cependant l'ensemble de ces travaux ne traite pas de l'évolution des formes et des intensités d'engagement avec ces ressources, en fonction de la manifestation des troubles. Cela s'explique par la prédominance d'analyses menées dans des environnements institutionnels, occultant les pratiques dans l'espace urbain du quotidien et en particulier dans l'espace public (McGrath et Reavey, 2016; Parr, 1999). Par ailleurs, ces études se focalisent sur les moments de manifestation aiguë des troubles, ce qui ne permet pas de saisir l'évolution des formes d'engagement au fur et à mesure du rétablissement. La plupart de ces études considère en outre le rétablissement dans une perspective linéaire qui se caractérise par un lent processus de stabilisation des troubles. Le caractère fluctuant et aléatoire des troubles au cours de ce processus est en revanche peu traité par cette littérature, alors même qu'il constitue un élément caractéristique du rétablissement des personnes souffrant de troubles de la schizophrénie (Klausner, 2014; Corin, 2002).

De plus, à l'image de la littérature en géographie de la santé mentale, il existe également un flou autour du diagnostic des populations étudiées dans la plupart de ces recherches, puisque celles-ci ne distinguent pas les expériences psychotiques chroniques de celles survenant occasionnellement.

\subsection{L'intensité d'engagement de Laurent Thévenot}

Le sociologue Laurent Thévenot développe ce qu'il nomme «une sociologie de la raison pratique», dans laquelle il considère l'individu et son action à travers ses engagements multiples dans le temps et dans l'espace (Thévenot, 2006 : 92). Ces engagements sont à l'origine des différentes relations qu'un individu tisse avec son environnement dans la réalisation de son action. Cette dernière n'est cependant pas le résultat d'une intention bien formée. Elle se définit plutôt comme une succession de formes variées de coordination avec l'environnement, évoluant au fil des situations et des circonstances. Chaque action se traduit par une forme d'engagement avec l'environnement qui s'ajuste en fonction de la portée de l'action. Cet engagement se décline à différentes intensités. Afin de pouvoir mieux les caractériser, Thévenot développe le concept «d'intensité d'engagement» (Thévenot, 2006). Celui-ci désigne le type de rapport développé avec l'environnement dans la réalisation d'une action. En fonction de sa portée, la relation à l'environnement requiert une coordination et un ajustement plus ou moins étroits avec les pratiques des autres.

Si la portée de l'action est publique, les exigences de coordination avec l'environnement social s'accroissent, de même que l'intensité de la relation, impliquant une compréhension des pratiques des autres acteurs et une coordination avec celles-ci dans la réalisation de l'action. À l'inverse, si la portée de l'action est plus personnelle, les exigences de coordination se réduisent, de même que l'engagement avec l'environnement.

Dans le cadre de mon analyse, le concept «d'intensité d'engagement» proposé par Thévenot (2006) me sera utile pour rendre compte avec précision du degré d'investissement au cours des pratiques de retrait et de reconquête du rétablissement.

Ce concept offre la possibilité de détailler les différentes formes d'engagement personnelles et collectives inhérentes aux pratiques de rétablissement des jeunes gens. À cet effet, je distingue les formes d'engagement développées lors des pratiques de retrait, des formes d'investissement propres aux pratiques de reconquête de l'espace urbain.

\section{Méthodologie}

Dans le cadre de cette recherche, je m'appuie sur une ethnographie du quotidien que j'ai menée avec de jeunes personnes souffrant de schizophrénie d'avril à juillet 2016 dans une institution thérapeutique lausannoise. Celle-ci est située au coeur de Lausanne, dans un des quartiers les plus denses 
de la ville ${ }^{9}$. Le quartier de l'institution, très accessible en transports publics, abrite des logements, des commerces, des bureaux, des infrastructures publiques (écoles, piscine, hôpital) mais aussi des espaces de loisirs, tels que des parcs publics urbains. L'institution possède plusieurs locaux d'activités et de soins ainsi qu'une dizaine d'appartements protégés, tous situés dans ce même quartier.

Pour comprendre l'ancrage de l'institution dans le quartier, il faut remonter à sa création en 1955 sur une initiative privée (Chevalley, 2016). Cet espace est précurseur de la période de désinstitutionalisation de la psychiatrie en Suisse. L'institution offre une alternative à l'asile à une époque où les longs séjours à l'hôpital psychiatrique sont encore une pratique courante partout en Europe, de même qu'en Suisse (Chevalley, 2016). Le choix de localisation de cet espace n'est pas le fruit du hasard. Il correspond à la vision intégrative du propriétaire de l'institution de l'époque qui souhaitait trouver un espace situé en ville, afin de faciliter l'intégration des personnes souffrant de troubles psychiques à l'échelle du quartier. La vision préconisée était celle de l'espace urbain comme un instrument de soins à part entière. D'un lieu de vie, l'institution se mue au fil des années, en un hôpital de jour qui inclut un suivi thérapeutique, des activités de groupe, de la réhabilitation professionnelle et des possibilités de logement pour ceux qui en ont besoin (Chevalley, 2016). L'objectif de l'institution est d'accompagner les jeunes gens dans leur processus d'autonomisation à travers des activités individuelles et collectives qui ont lieu dans et hors de l'institution.

Les participants appartiennent tous au programme lausannois de traitement et d'intervention dans la phase précoce des troubles psychotiques (TIPP). Ce programme a été mis en place par le Département de psychiatrie du centre hospitalier universitaire vaudois (CHUV) en 2004. L'ensemble des participants a vécu un premier épisode psychotique remontant à moins de trois ans et est âgé entre dix-huit et trente ans ${ }^{10}$.

Ces participants sont au nombre de seize. Ils sont tous originaires de Suisse romande. La moitié d'entre eux viennent de la région lausannoise. Avant leur admission à l'institution, les participants connaissaient peu ou mal ce quartier de Lausanne. C'est au cours des mois passés à l'institution que les jeunes gens ont développé une connaissance approfondie du secteur. L'ensemble des participants à mon étude fréquente l'institution depuis au moins trois mois et a développé un lien de familiarité avec plusieurs espaces situés à proximité de l'institution. Quatre des participants interrogés

\footnotetext{
${ }^{9}$ Données issues des statistiques de la ville de Lausanne basées sur les données de 2017 (source : https://www.lausanne.ch/ statistique (la date du dernier accès : 14 novembre 2018), fiche de population, juin 2018).

${ }^{10}$ Dans le cadre de cet article, les participants à l'étude ont vécu un premier épisode psychotique datant de moins de trois ans. Cependant, au moment de mon travail de terrain, la plupart d'entre eux a déjà connu des périodes de rechutes, se traduisant parfois par le vécu d'un ou de deux autres épisodes.
}

vivent, par ailleurs, en appartement protégé depuis plus d'une année dans ce même secteur, ce qui participe également à développer des liens de familiarité avec le quartier.

Dans le cadre du terrain ethnographique, $\mathrm{j}$ 'ai pris part au quotidien de l'institution thérapeutique pendant une période de trois mois. Tout au long de cette période, j'ai participé aux différentes activités thérapeutiques organisées dans et hors institution. Grâce à ma présence régulière, j'ai aussi pu m'impliquer dans l'organisation de la vie de l'institution à laquelle prend part l'ensemble de l'institution. J'ai aussi participé aux tâches et aux réunions réservées au personnel soignant de l'institution. J'ai également été amenée à côtoyer certains participants et soignants dans des activités quotidiennes réalisées hors de l'institution pendant la journée. Dans le cadre de mon terrain, j'ai privilégié la méthode d'observation participante car elle permet de pallier les difficultés dialogiques et verbales rencontrées par les participants, caractéristiques des personnes souffrant de troubles psychotiques (Davidson, 2003 ; Lysaker et Lysaker, 2008).

Cette démarche ethnographique a été l'occasion de m'immerger dans le quotidien des jeunes. J'ai ainsi pu partager des expériences avec eux in situ. Grâce à cet accès immédiat à leur expérience, j'ai pu observer leur vécu des troubles et leurs pratiques urbaines dans les moments de manifestation des symptômes. Ma présence quotidienne à l'institution et le partage d'expériences communes m'a permis de progressivement tisser des liens avec les participants et de gagner leur confiance.

Tout au long des trois mois de terrain, j'ai suivi les diverses étapes propres à une démarche ethnographique (Lapassade, 1993). J'ai d'abord rigoureusement et quotidiennement consigné par écrit mes observations, de même que les diverses discussions informelles avec participants et soignants dans un journal de bord. Cela m'a permis de répertorier de façon systématique les différentes données de terrain récoltées au fur et à mesure de mon ethnographie.

Au cours du dernier mois de mon expérience de terrain, j'ai procédé à de premières analyses spontanées sur la base des données déjà récoltées. J'ai ainsi pu formuler une première série d'observations et une proposition de classification des données sur la base des différents faits relevés. J'ai ensuite confronté ces premières analyses aux remarques et aux critiques des soignants et des participants dans le cadre de groupes de discussion ${ }^{11}$. Cette démarche de confrontation a été répétée régulièrement au cours du travail de terrain, permettant ainsi d'évaluer et de réorienter, si nécessaire, les premières analyses et les tentatives de classification des données.

Après la phase de terrain, sur la base de ce premier travail d'analyse, j'ai procédé à une classification systématique des transcriptions de mon ethnographie. J'ai constitué des catégories thématiques permettant d'ordonner les données

\footnotetext{
${ }^{11}$ Les groupes de discussion sont aussi communément appelés «focus groups discussions » en anglais (Hennink, 2014).
} 
de manière cohérente, complète, logique et succincte. Sur la base de cette classification, $j$ ' ai pu identifier et formuler une série de concepts pertinents dans le cadre de mon ethnographie. Enfin, à la fin de mon ethnographie, j'ai complété mon analyse par cinq entretiens menés respectivement avec des participants et des soignants. Cela m'a permis d'obtenir des éléments de détails ou des précisions supplémentaires nécessaires à la compréhension du fonctionnement de l'institution ainsi qu'à certaines pratiques observées.

Dans cette optique, les résultats qui font l'objet de la section suivante se basent sur des observations récurrentes et systématiques qui ont émergé tout au long de mon ethnographie. Les expériences des participants que je cite et que je développe dans mon analyse se réfèrent à des situations-types rencontrées tout au long de mon ethnographie avec d'autres jeunes de l'institution.

\section{Résultats}

Les observations de terrain révèlent trois aspects centraux des pratiques du rétablissement. Tout d'abord, les temporalités de rétablissement se caractérisent par une logique cyclique (Baumann et al., 2019). L'analyse met ensuite en évidence l'existence de deux cycles de rétablissement, l'un court et l'autre long qui correspondent respectivement aux courtes périodes de manifestation des troubles et à la période d'atténuation des troubles plus ou moins durable.

Le deuxième aspect mis en évidence par les résultats a trait à l'extension du périmètre des pratiques de rétablissement des jeunes gens et à leur reconquête progressive de la ville, au fur et à mesure de la rémission des troubles. Je montre plus spécifiquement que cette reconquête s'effectue depuis des lieux-refuges, tant des espaces publics que des lieux de soins institutionnels, vers d'autres environnements urbains moins familiers.

Troisièmement, les résultats montrent que les participants modulent leurs pratiques de retrait ou au contraire de reconquête en fonction des cycles courts et longs, ce qui se traduit par une mobilisation variable des diverses ressources et par l'activation de «prises » offertes par l'environnement (Golembiewski, 2013). À ce titre, je propose une typologie de ressources d'ordre matériel, social et sensoriel mobilisées par les participants au cours de ces deux cycles du rétablissement.

En dernier lieu, je mets en évidence que le degré d'engagement des participants avec leur environnement augmente au fur et à mesure de la rémission des troubles.

\subsection{Temporalités des troubles psychotiques}

Les observations de terrain révèlent tout d'abord le caractère fluctuant et cyclique des troubles au cours du rétablissement. Les participants m'expliquent que les troubles interviennent de façon fluctuante mais récurrente à différents moments de la journée, à l'image de l'expérience de Nathan :
«Au cours des semaines passées à l'institution, j'observe que Nathan s'absente régulièrement pendant ou après les activités pendant la journée. Un jour, alors que nous prenons un café, je demande à Nathan la raison de ces absences. Il réfléchit un instant, puis m'explique : “Quand ça va pas bien là-haut (Nathan montre sa tête), je pars tout de suite de l'institution. J'entends tout d'un coup des voix ... elles me disent quoi faire. Et là avec tout le bruit autour de moi, l'agitation ... ça fait trop ... Dans ces moments-là, je préfère m'isoler et attendre que ça passe. Quand ça s'arrête, je rentre à l'institution. Il y a quelques mois j'ai eu une crise ... c'était horrible ... je suis sorti de l'hôpital... je restais chez moi, j'avais tout le temps des voix qui me parlaient. Maintenant, même si ça revient encore ... Il y a des jours où ça va mieux et au moins ... j'arrive à sortir" » (Extrait du cahier des observations, juillet 2016).

Dans cet extrait, Nathan explique en effet que les troubles surviennent de façon brève à différents moments de la journée de façon fluctuante mais récurrente, à la façon d'un cycle. Mais depuis sa sortie d'hospitalisation, le jeune homme remarque qu'avec le temps, le rythme et l'intensité des troubles s'atténuent progressivement. Ces fluctuations de rythme d'apparition chez les jeunes constituent une réalité quotidienne à laquelle sont confrontés les soignants de l'institution et avec laquelle ils doivent composer, comme l'explique Géraldine, membre de l'équipe soignante : «[...] On sait que chez les jeunes qui souffrent de schizophrénie on peut avoir un changement brutal de comportement... comme ça au cours de la journée... souvent dû à la réapparition de symptômes ... on les accompagne dans ces moments difficiles ... ça fait partie de notre quotidien ici » (Extrait du cahier des observations, juillet, 2016).

Ensuite, ces observations attestent de l'existence de deux temporalités différentes du rétablissement qui correspondent à ce que j'ai nommé le cycle long et le cycle court. Nathan explique en effet qu'au cours du lent processus de stabilisation des troubles après sa sortie d'hôpital - qui correspond au cycle long -, il entend à intervalles réguliers des voix dans sa tête. Celles-ci sont l'expression temporaire des symptômes et elles correspondent au cycle court. Ces cycles sont caractéristiques de la période de rétablissement de la schizophrénie et ils interviennent chez de nombreux participants, comme en témoignent les propos d'Aline, membre de l'équipe soignante qui travaille dans l'institution depuis une dizaine d'années : «Avec les collègues on sait que... les jeunes ... quand ça va mal... ils ont le réflexe de chercher refuge dans un endroit isolé, souvent ils restent d'ailleurs chez eux quand ils sentent qu'il y a une aggravation dans leur souffrance psychique. Surtout après les hospitalisations c'est toujours très difficile... Nous on les encourage quand même à venir même quand ça va pas... c'est important qu'ils ne s'enferment pas dans leur souffrance. Ensuite... on observe souvent une amélioration... après quelques jours ... quelques semaines ... ou quelques mois ... quand la crise est passée... on les revoit 
ici. Mais je dirai que cette souffrance est toujours présente chez chacun des jeunes, simplement elle s'aggrave par moments et chez certains elle est plus grande et elle dure plus longtemps... » (Extrait du cahier des observations, juillet 2016).

Le récit d'Aline confirme que ces deux cycles constituent le quotidien des jeunes participants. Comme l'explique la soignante, les troubles interviennent toujours ponctuellement et de façon récurrente chez les jeunes participants. Puis, ils s'atténuent ou disparaissent temporairement, ce qui permet aux participants de reprendre normalement le cours de leur vie. Chez certains participants, le cycle long dure plus longtemps que chez d'autres, comme le souligne Aline.

Un autre cas emblématique des situations vécues par plusieurs participants récemment sortis d'hospitalisation est celui d'Éléonore, une jeune femme qui fréquente l'institution depuis deux ans. Lors d'une discussion avec cette jeune participante, j'apprends que depuis son arrivée elle effectue régulièrement des séjours à l'hôpital en raison de la régularité et de la forte intensité de ses troubles. Il arrive en effet chez certains participants, comme c'est le cas pour Eléonore, que les troubles persistent et apparaissent de façon récurrente tout au long de la vie. Chez d'autres participants, le rythme d'apparition des troubles se stabilise, puis s'atténue progressivement, jusqu'à la disparition complète des symptômes, comme c'est le cas de Mathys. Ce jeune participant est resté seulement quelques mois à l'institution. Avant son départ il m'explique : «Je me sens de nouveau comme avant et ces quelques mois ... ce qui m'est arrivé... C'était comme une parenthèse ... heureusement c'est fini maintenant» (Extrait du cahier des observations, juillet 2016). Dans la majorité des cas, les troubles des jeunes disparaissent ou du moins s'atténuent permettant ainsi le retour à une vie quotidienne ordinaire, comme en témoigne cette discussion avec l'équipe soignante : «C'est une grande victoire pour le jeune... mais pour nous aussi... (rire) quand on arrive à l'accompagner pour qu'il puisse retrouver une vie à peu près normale ... un quotidien comme avant... d'ailleurs la majorité des jeunes que nous suivons s'en sortent et on ne les revoit plus...» (Extrait du cahier des observations, focus group, juin 2016).

\subsection{Du retrait à la reconquête urbaine}

Dans la suite de l'analyse, je mets en évidence l'extension du périmètre de pratiques des participants et le processus de reconquête de l'espace urbain, au fur et à mesure de la rémission des troubles. Je montre que le périmètre de pratiques se restreint lors de la manifestation des troubles propre au cycle court. À l'inverse, les participants étendent leurs pratiques à d'autres espaces, au fur et à mesure de la rémission des troubles, caractéristique du cycle long.

Le travail propose ensuite une typologie des ressources matérielles, sociales et sensorielles mobilisées par les participants dans leurs pratiques de retrait et de reconquête. Il s'agit aussi de faire ressortir l'évolution de l'usage de ces ressources en fonction du cycle court et du cycle long. Enfin, l'analyse démontre que le nombre et la diversité de ressources utilisées par les participants permettent de renseigner le degré d'engagement de ceux-ci avec leur environnement.

\subsubsection{Un retrait de l'environnement}

Lors du cycle court, marqué par une manifestation récurrente des troubles, les participants restreignent le périmètre de leurs pratiques. De façon générale, les jeunes gens se déplacent dans un périmètre circonscrit qui s'organise autour d'espaces connus, qu'ils fréquentent habituellement au quotidien. Ces lieux leur procurent un sentiment de sécurité et de sérénité dans les périodes de crise (Klausner, 2014 ; Corin, 2002 ; Parr, 1999). À l'image de Raoul, un jeune homme de vingt-deux ans récemment arrivé à l'institution, lors des intenses moments de souffrance, les participants se retirent dans des espaces familiers, loin de l'activité sociale. Ces périodes s'accompagnent souvent d'une perte de repères spatiaux et sociaux : «Quand je suis sorti d'hospitalisation, j'avais besoin d'être dans des endroits que je connaissais et avec des gens que $\mathrm{j}$ 'appréciais ... qui sont proches ... tu vois ... la crise ça te change toutes tes perceptions ... tu ressens tout de façon très intense... alors si tu es dans un endroit que tu connais pas ou mal... ça peut très vite devenir hyper-stressant et angoissant... du coup après tu recommences à réfléchir trop... et pis tu repars dans des délires ... » (Extrait du cahier des observations, juin 2016). Les participants cherchent refuge dans des espaces, à l'échelle du quartier, à proximité ou au sein de l'institution thérapeutique et qui permettent de limiter et d'éviter les interactions sociales et sensorielles avec leur environnement. Comme l'explique Raoul, les pratiques de retrait sont liées à une augmentation significative de la sensibilité à l'environnement au moment de la manifestation des troubles (Kapur, 2003). Ce phénomène est en effet récurrent chez les personnes souffrant de troubles de la schizophrénie. L'intensification de la sensibilité à l'environnement mène souvent à un phénomène de surstimulation sensorielle au cours duquel les personnes ont l'impression d'être submergées par les sollicitations rencontrées dans l'environnement (Kapur, 2003; Myin-Germeys et al., 2009). Les participants mettent ainsi à distance les stimulations de l'environnement. Ils cherchent avant tout à éviter le bruit et les interactions sociales qui sont susceptibles d'aggraver les symptômes et de provoquer une surstimulation sensorielle, et ceci jusqu'à l' atténuation et jusqu'à la disparition des troubles.

Les pratiques de retrait et de mise à distance de l'environnement que j'ai pu observer au cours de mon travail, ont également été constatées par l'équipe soignante et notamment, par Martine une thérapeute, qui m'explique que les soignants doivent gérer ces pratiques au quotidien : «[...] souvent on doit téléphoner chez les participants, le matin, après l'appel... il y a en a beaucoup qui juste après l'hospitalisation n'arrivent pas à venir parce qu'ils se sentent mal 
ou fatigués... on voit souvent une période pendant laquelle ils se retirent de tout ... ils évitent beaucoup de choses... Du coup, on les appelle pour savoir comment ils vont. En fonction de leur état, on leur propose de venir au moins quelques heures ici ... » (Extrait du cahier des observations, entretien, mai, 2016).

Dans cette perspective, les observations montrent qu'une partie des participants s'approprie essentiellement les espaces institutionnels de soins qui se composent principalement de l'institution thérapeutique, de l'hôpital et des centres de consultation ambulatoires. Les participants fréquentent essentiellement les espaces de soins et ils privilégient la proximité du personnel médical qui leur procure un sentiment de sécurité, comme l'explique Eléonore : «Quand je suis en crise... je préfère être chez moi ou à l'institution... ce sont des endroits où je me sens en sécurité et que j'ai l'habitude de fréquenter... à l'institution il y a des professionnels de la santé qui peuvent m'aider si ça va pas... chez moi... c'est ma mère qui m'amène aux urgences... ils me connaissent là-bas... quand $j$ 'arrive ils savent quoi faire... » (Extrait du cahier des observations, juillet 2016). Plusieurs participants cherchent exclusivement refuge dans des espaces intérieurs et confinés qui leur confèrent protection et tranquillité, à l'image de Dan, un jeune homme de vingt-quatre ans qui fréquente l'institution depuis trois mois : "Juste après mon hospitalisation... j'avais beaucoup de peine à être dehors ... maintenant ça va mieux ... mais j'avais besoin d'être dans un endroit circonscrit, dedans...» (Extrait du cahier des observations, juin 2016).

De même, le lieu d'habitation constitue également un refuge familier en retrait de l'activité sociale. À l'image d'Eléonore, les participants privilégient également leur lieu d'habitation, car il offre la possibilité d'un retrait momentané et permet d'atténuer la souffrance des jeunes gens. À l'instar de Liam et Mélusine, deux jeunes participants de vingt ans qui fréquentent l'institution depuis quelques mois, les jeunes gens préfèrent en général rester chez eux, avec leur famille, lorsque les symptômes se manifestent, au cours d'une période de rechute. Cela leur permet en effet de se remettre de leur souffrance dans un environnement qui leur procure confort et bien-être : "[... ] quand on n'est pas bien ... je sens quand ça commence... on peut se reposer loin du bruit et des autres de l'institution, de toute l'activité qui est très pénible à supporter, en attendant que ça aille mieux" explique Liam. Mélusine hoche la tête et ajoute : "oui, c'est exactement ça. Et puis chez moi, il y a aussi ma mère qui est là au cas où ça va pas. Elle est là pour moi. Si ça va pas elle appelle un médecin ou elle m'emmène aux urgences" (Extrait du cahier des observations, mai 2016).

Toutefois, à l'image de Nathan, certains participants fréquentent aussi des espaces publics familiers tels que des parcs, des cafés ou des commerces de proximité. Ces lieux sont tous situés à l'échelle du quartier et à proximité immédiate de l'institution. Cette proximité confère un senti- ment de sécurité aux participants, comme l'explique Nathan : «Les endroits où je vais quand je me sens mal sont dans le quartier... je pense qu'ils sont à cinq minutes à pied de l'institution... pas plus. Comme ça, si j'ai un problème, je peux retourner directement voir Aline [soignante en charge du suivi de Nathan]» (Extrait du cahier des observations, juillet, 2016).

À l'exemple du jeune Raoul âgé de vingt-quatre ans et depuis deux ans à l'institution, d'autres participants fréquentent les cafés du quartier, des lieux dans lesquels Raoul se sent « comme chez lui ... toujours bien accueilli» (Extrait du cahier des observations, juillet, 2016). Ces lieux sont considérés comme familiers par Raoul, car il connaît les propriétaires des cafés qui le laissent lire son journal dans un coin de la salle, autant de temps qu'il le souhaite.

De même, les participants fréquentent les espaces verts à proximité de l'institution, à l'image de Nathan qui se rend quotidiennement dans le parc urbain situé à deux pas de l'institution. Les parcs et les forêts sont en effet connus pour leurs propriétés thérapeutiques dans les soins des troubles psychiques (Curtis, 2010; McGrath et Reavey, 2016). Les espaces verts permettent par exemple de réduire l'anxiété et de développer un sentiment de sécurité (De Vries et al., 2003). De même, ces espaces agissent parfois également comme médiateurs dans les situations stressantes vécues par les personnes souffrant de troubles psychiques, en limitant les sollicitations de l'environnement vécues comme désagréables et ils permettent d'atténuer et de limiter les sollicitations de l'environnement (Evered, 2016). Ainsi, ce sont véritablement les propriétés calmantes et tranquillisantes des espaces verts qui aident les participants à surmonter les moments de crise au cours de leur rétablissement, comme en témoignent ces observations : « Lors d'une activité organisée par l'institution dans un parc, je demande aux jeunes gens de me décrire ce qui leur plaît dans cet espace. Ils m'expliquent tous que ces lieux aident à se calmer quand on n'est pas bien, à réfléchir sur soi, à prendre de la distance avec sa vie et pour certains à méditer» (Extrait du cahier des observations, juin, 2016).

Ces espaces se situent donc dans un périmètre circonscrit, à proximité de l'institution thérapeutique. Lors du cycle court, les participants privilégient essentiellement des pratiques de mise à distance de l'environnement qui impliquent la mobilisation de ressources matérielles, sociales et sensorielles spécifiques.

\subsubsection{Une mobilisation de ressources matérielles, sociales et sensorielles familières}

Lors du cycle court, les participants ne s'isolent pas de toute interaction avec leur environnement. Les observations montrent qu'ils conservent un lien privilégié avec les ressources familières offertes par celui-ci (Duff, 2012). Les jeunes gens mobilisent des ressources matérielles, sociales et sensorielles qui font partie de leur quotidien et qu'ils ont l'habitude d'utiliser. Ces ressources permettent d'apporter du 


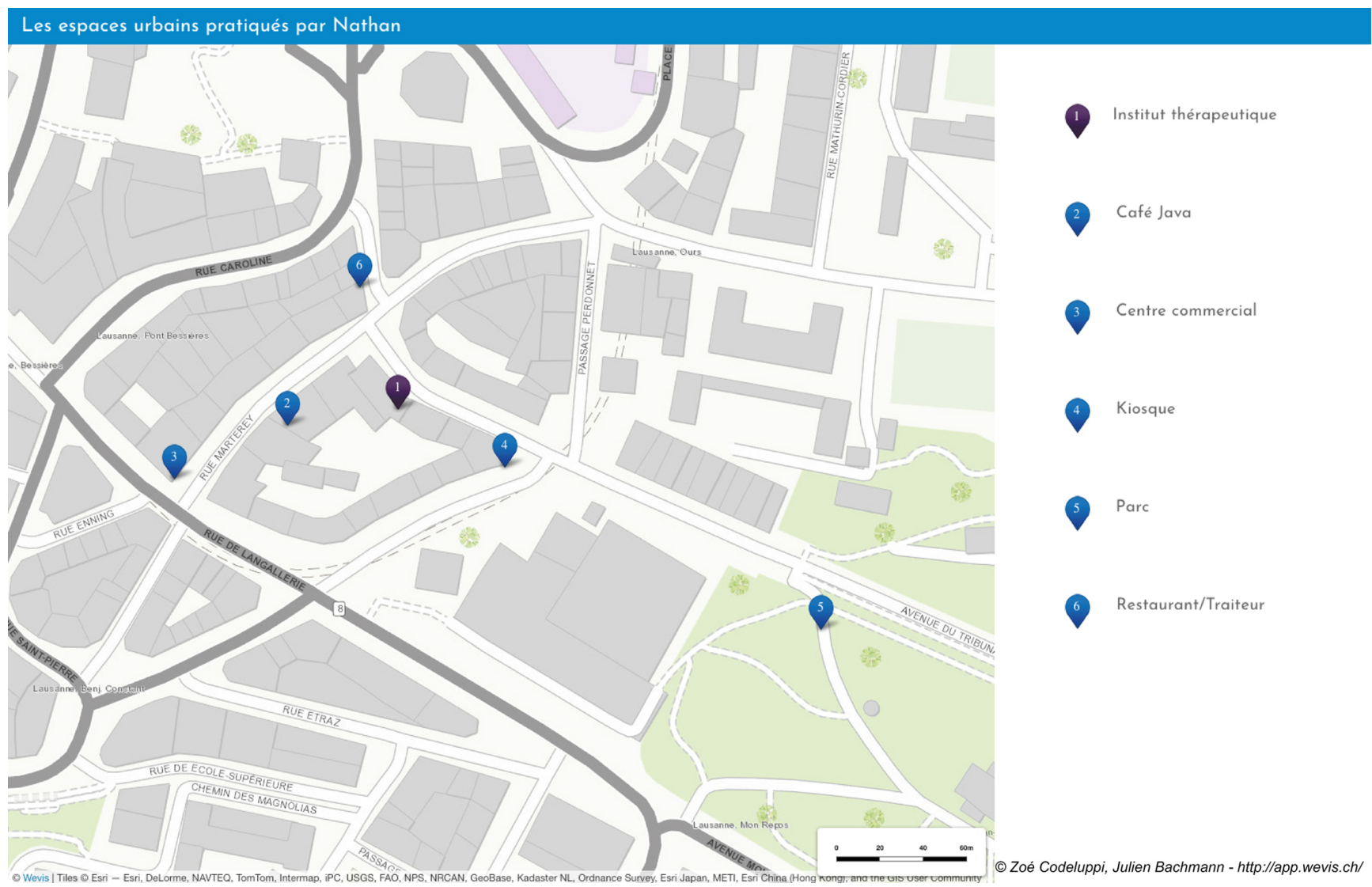

Figure 1. Les espaces familiers de retrait de Nathan.

confort et un sentiment de sécurité, mais aussi d'apaiser la manifestation des troubles (Duff, 2012, 2014). Dans la suite de l'analyse, je propose une typologie des ressources familières matérielles, sociales et sensorielles qui sont employées par les participants dans le cadre de leurs pratiques de retrait.

Premièrement, les participants mobilisent des ressources matérielles spécifiques. Celles-ci regroupent l'ensemble des objets utilisés par les participants ainsi que les caractéristiques matérielles d'un espace, comme la présence de végétation ou de mobilier urbain (Duff, 2014). J'observe que les participants amènent très souvent avec eux des objets personnels, comme des livres, mais aussi des téléphones et des ordinateurs portables qu'ils mobilisent à l'institution, mais également à l'extérieur, dans les espaces publics. Ces objets embarqués constituent de véritables «prises » dans l'environnement (Golembiewski, 2013; Duff, 2014) qui leur permettent de médiatiser les sollicitations de l'environnement et de surmonter les moments de mal-être, survenant avec la manifestation des troubles. Marianne par exemple, une jeune participante âgée de vingt-trois ans qui fréquente l'institution depuis une année, utilise la lecture pour surmonter ses troubles :

«Marianne apporte souvent des lectures lors de ses journées à l'institution. Lors des pauses, elle s'installe la plu- part du temps dans la salle principale, dans un coin, en train de lire un livre. Elle m'explique que la lecture, surtout les livres "philosophiques" l'aident à "surmonter les moments difficiles" qu'elle vit actuellement avec sa "maladie". "Je ne sors pas de chez moi sans un livre dans mon sac" me dit-elle en rigolant » (Extrait du cahier des observations, juin 2016).

De même, les objets électroniques tels que les téléphones ou les ordinateurs portables permettent aux participants de médiatiser leurs troubles et d'apporter distraction et réconfort dans les instants de souffrance, à l'image de Paul, un jeune participant de vingt-quatre ans, qui fréquente l'institution depuis 9 mois : «Paul apporte régulièrement son ordinateur portable. Il s'installe pendant de longues heures dans la bibliothèque de l'institution, à l'écart des autres. Il m'explique qu'il aime bien "travailler" sur son ordinateur. Il aime surfer sur internet, apprendre de nouvelles choses, échanger avec des amis sur les réseaux sociaux. Cela lui permet, me dit-il, de se distraire et de penser à autre chose lorsqu'il n'est pas suffisamment "en forme pour participer aux activités comme les autres" » (Extrait du cahier des observations, mai 2016).

La fonction des objets électroniques dans le rétablissement des participants est d'ailleurs souvent discutée lors de réunions au sein de l'équipe soignante. Ces objets électroniques sont souvent qualifiés de «doudous affectifs» par 
l'équipe soignante, compte tenu du lien affectif fort que les participants développent avec ces médiateurs qui leur apportent «beaucoup de réconfort et un sentiment de sécurité » (Extrait du cahier des observations, focus group, juillet, 2016).

Les participants privilégient également des configurations spatiales particulières dans les moments de souffrance. À l'instar d'Eléonore, Dan, Liam ou encore Mélusine, évoqués précédemment, les jeunes gens privilégient des espaces intérieurs, de petite dimension et bien circonscrits. Ces configurations confèrent aux participants un sentiment de sécurité et de maîtrise de leur environnement.

Pour d'autres participants comme Nathan, les espaces publics familiers, tout particulièrement les lieux végétalisés pourvus de mobilier urbain, comme les parcs permettent de médiatiser leurs troubles : "Souvent je vais au parc à côté de l'institution. J'aime m' asseoir sur les bancs et regarder les arbres et la vue sur le lac. Il y a aussi un coin ... vers une fontaine où je vais souvent... j'aime bien entendre le bruit de l'eau ... ça me calme» (Extrait du cahier des observations, juillet, 2016). Le son de l'eau est souvent apprécié par les participants car il permet d'atténuer le vécu des troubles.

La configuration du parc offre ainsi des «prises » qui procurent du bien-être à Nathan (Golembiewski, 2013). Le rôle ressourçant des lieux de nature fait l'objet de discussions au sein de l'équipe soignante. Martine m'explique : «[...] quand il y a des sorties organisées par l'institution ... les patients réclament... ils veulent aller dans des parcs, au bord $\mathrm{du}$ lac ou parfois dans la forêt... ils aiment bien se poser dans ces endroits... il y a vraiment un effet thérapeutique évident» (Extrait du cahier des observations, juillet, 2016).

Deuxièmement, les participants privilégient les ressources sociales familières qui leur procurent un sentiment de sécurité. Cela se traduit par la présence, la proximité et la disponibilité de personnes proches des jeunes gens ou du personnel soignant. À l'image de Mélusine, citée plus haut, qui privilégie la proximité avec sa mère, lors de la manifestation temporaire des troubles, les jeunes gens préfèrent interagir avec des proches et des personnes de confiance qu'ils connaissent bien et qui leur permettent de surmonter leur moment de souffrance. D'autres participants comme Éléonore privilégient le contact avec le personnel soignant et médical de l'institution, comme elle me l'explique lors d'une conversation : «[...] ce sont des personnes de confiance qui me connaissent bien et avec qui je peux parler quand il y a des moments difficiles. En plus, ils sont toujours là pour moi ... quand j' ai besoin. Ils savent aussi quoi faire si je me sens mal. Dans ces momentslà, j'aime pas trop être avec d'autres gens de l'institution que je connais moins... ça me stresse » (Extrait du cahier des observations, juin 2016).

À l'instar de Raoul, d'autres participants préfèrent se rendre dans des cafés de quartier, dans lesquels ils créent un petit espace de sociabilité et d'échange avec des personnes de leur entourage et des connaissances à même de comprendre ces moments de souffrance et qui, par leur pré- sence et par leur empathie, les aident à surmonter ces périodes difficiles : «Quand je vis des moments difficiles, je vais au café... je connais bien le patron... il m'accueille toujours ... il me laisse un petit coin tranquille... et quand il voit que ça va pas bien ... ben ... il s'assied à côté de moi et il m'écoute parler... des fois je préfère rester seul... du coup... il m'amène le journal, le café et il me dit toujours : "Si tu as besoin de quelque chose Raoul, on est là, ok?" (rires). C'est vraiment un endroit où je me sens bien làbas ... mieux qu'à l'institution ... en fait ... » (Extrait du cahier des observations, juin 2016).

Enfin, les ressources sensorielles mobilisées par les participants lors du cycle court sont peu nombreuses. Comme mentionné plus haut, ceux-ci évitent toute sollicitation sensorielle en raison du phénomène de surstimulation sensorielle déjà évoqué plus haut (Kapur, 2003). Les jeunes gens cherchent à se préserver du bruit ainsi que de toute interaction avec des inconnus qui amplifient leur mal-être au moment de la manifestation des troubles (Söderström et al., 2017). Eléonore, par exemple explique : «Dans ces moments-là [les moments de crise] ... j'ai beaucoup de peine avec le bruit... avec les gens... surtout ceux que je connais pas... la proximité physique aussi... c'est problématique pour moi ... après je peux très vite paniquer... » (Extrait du cahier des observations, juillet, 2016). Les jeunes gens privilégient ainsi les espaces calmes et en retrait de l'activité sociale, loin des stimulations auditives, visuelles et olfactives. La recherche de calme leur permet de retrouver un certain «calme intérieur» m'explique Eléonore et leur apporte davantage de bien-être (Extrait du cahier des observations, juillet 2016). Parfois, à l'instar de Nathan, dans les moments de crise, certains participants aiment fréquenter les parcs, car ils offrent des sollicitations auditives tels que les chants des oiseaux ou le son du vent dans les arbres qui sont vécues comme agréables et apaisent leurs souffrances.

\subsubsection{Un engagement limité avec l'environnement}

Les observations montrent que lors du cycle court, les participants s'engagent peu avec les ressources de leur environnement. Ils limitent le nombre et la variété de ressources mobilisées dans les périodes de manifestation des troubles (Thévenot, 2006). Dans ces moments-là, les jeunes gens mobilisent essentiellement des ressources matérielles et sociales familières, à l'image de Nathan, d'Eléonore et des autres participants cités précédemment. Ces ressources familières apportent en effet du réconfort aux individus et les aident à surmonter les périodes de crise.

Toutefois, de manière générale, les participants mettent à distance leur environnement ainsi que les ressources qui s'y trouvent et qu'ils maîtrisent moins. De même, ils évitent les espaces qu'ils connaissent mal. Les participants modulent ainsi leur degré d'engagement avec les ressources de l'environnement en fonction de la manifestation des troubles. La mise à distance de l'environnement se traduit notam- 
ment par la création de «bulles de protection» par les participants, leur permettant ainsi de se protéger des stimulations sociales et sensorielles, susceptibles d'aggraver leur ressenti des troubles (Söderström et al., 2017). Ces bulles agissent ainsi comme des filtres qui participent à limiter le degré d'engagement avec leur environnement et avec ces ressources peu familières. À cet effet, les objets électroniques sont utilisés par les participants pour filtrer leurs interactions avec l'environnement. Ils facilitent ainsi les pratiques de retrait temporaire de la part des participants, comme le montre l'exemple de Tom : «Pendant les moments de pauses, j'observe qu'une majorité de participants sont sur leurs téléphones et que quelques-uns pianotent sur leurs ordinateurs. J'observe notamment Tom, qui regarde des vidéos sur son téléphone, écouteurs dans les oreilles. Souvent, lors d'activités, il s'assied dans un coin et je l'entends régulièrement rigoler au fond de la salle. Un jour, après l'activité de danse, j'interroge Charlène, une soignante qui travaille depuis une dizaine d'années au sein de l'institution, sur les pratiques de Tom. Elle m'explique que Tom communique difficilement en raison de la gravité de ses troubles de la schizophrénie. La seule façon pour lui d'être présent lors des activités est de s'asseoir dans un coin de la salle, de préférence à côté d'une fenêtre, et de regarder des dessins animés. De cette façon, il "arrive à s'isoler dans une bulle et à rester avec les autres en se concentrant sur autre chose" me dit-elle » (Extrait du cahier des observations, mai, 2016). Grâce à ces pratiques, les participants se constituent une «bulle de protection» qui leur permet de rester en retrait et de ne pas s'investir avec leur environnement social et sensoriel. En limitant leurs interactions avec l'environnement, les participants ne doivent pas fournir d'efforts pour se coordonner avec leur environnement social et matériel, contrairement aux pratiques de reconquête du cycle long.

Dans la suite du travail, je détaille les pratiques de reconquête ainsi que leurs spatialités, caractéristiques du cycle long du rétablissement.

\subsubsection{Une reconquête de l'espace urbain}

Le cycle long du rétablissement se caractérise par une extension progressive du périmètre des pratiques des participants. Ceux-ci reconquièrent en effet progressivement l'espace urbain, au fur et à mesure de la stabilisation ou de l'atténuation des troubles (Klausner, 2014). Le périmètre de pratiques ne se restreint plus désormais à l'échelle du quartier de l'institution, mais il s'élargit à l'ensemble de la ville. Comme l'explique Aline, membre de l'équipe soignante, chez la plupart des participants, la manifestation des troubles s'atténue ou se stabilise progressivement après quelques mois et elle s'accompagne d'un changement de spatialité de leur rétablissement : «La majorité des jeunes gens qu'on accueille arrivent à s'en sortir et retournent à leur vie d'avant ... On les accompagne après leur hospitalisation qui est souvent un moment difficile autant pour le jeune que pour les proches ... [...] au début ... ils ont beaucoup de mal à sortir de l'institution ... et puis après un moment... quand ça va mieux ... ils sortent en ville... ils récupèrent leurs anciennes habitudes ... jusqu'à ce que ce soit comme avant ... après quand ils vivent des moments de vulnérabilité qui souvent peuvent resurgir... mais pas toujours ... on les revoit à l'institution ... c'est leur lieurefuge en quelque sorte...» (Extrait du cahier des observations, juin, 2016). Ainsi, les participants se réapproprient progressivement l'espace urbain au fur et à mesure de leur rétablissement. Toutefois, comme le souligne Aline, au cours du processus de rémission des troubles, les participants sont souvent confrontés à des rechutes momentanées. Dans ces moments-là, les jeunes gens restreignent leur périmètre de pratiques et ils cherchent refuge dans des espaces familiers qui ont été décrits précédemment.

Cette reconquête s'opère à partir des espaces familiers qui servent de point de départ à une extension progressive de la réappropriation de la ville (Klausner, 2014). Patrick par exemple explique : «Au début, je préférais rester à l'institution... j'osais pas trop sortir... je me sentais pas assez bien... maintenant j'aime beaucoup aller dans des cafés au centre-ville... quand il fait beau... je m'assieds à une terrasse et j'observe les gens autour de moi... parfois j'échange quelques mots avec un autre client ou le serveur... c'est toujours très accueillant... il y a une bonne ambiance» (Extrait du cahier des observations, juin, 2016). Les espaces investis, lors de cette reconquête, sont essentiellement des lieux publics, comme des places ou des rues piétonnes du centre-ville, mais aussi des commerces et des cafés ou encore des bâtiments publics, tels que les églises, les bibliothèques ou les musées (Baumann et al., 2019). À l'instar de Patrick, les participants recherchent des espaces actifs et stimulants qui offrent de nouvelles ressources ou «prises» (Golembiewski, 2013). Les jeunes gens investissent, en particulier, les espaces publics qu'ils considèrent comme «accueillants», dans lesquels ils trouvent des opportunités d'inclusion sociale et de contact, de même qu'un niveau de stimulations sensorielles agréable (Curtis, 2010; Baumann et al., 2019). L'atténuation progressive du phénomène de surstimulation sensorielle, au fur et à mesure du processus du rétablissement, contribue à cette ouverture sociale et sensorielle (Kapur, 2003 ; Myin-Germeys et al., 2009).

De même, les bâtiments publics situés un peu partout dans la ville sont également investis par les participants. Ces espaces ne présentent toutefois pas les mêmes caractéristiques que les autres lieux. Ils sont en effet appréciés pour leur calme et pour l'absence de sollicitations sociales et sensorielles trop intenses (Codeluppi, 2016).

Ainsi, à l'image de Jim, certains participants privilégient des espaces peu actifs et peu stimulants socialement et sensoriellement : «[...] ça m'arrive souvent d'aller à la bibliothèque municipale et au Palais Rumine [musée et espace d'exposition temporaire]. J'aime bien cet endroit... c'est calme... je rencontre personne que je connais...j'aime bien me poser sur les tables à la bibliothèque ... ou en dehors 
et lire ou écouter de la musique... des fois je regarde aussi les expositions... » (Extrait du cahier des observations, juin 2016). Ces espaces offrent aux participants la possibilité de se reposer et ils leur confèrent un certain anonymat, propice au bien-être (Klausner, 2014).

Par ailleurs, à l'image de Nathan, les espaces publics comme les parcs sont très investis par les participants, tout au long du rétablissement : «[...] les parcs ... oui j'y vais très souvent ... quand ça va ... quand ça va pas aussi ... j'essaye d'y aller régulièrement... j'ai remarqué que ça me fait beaucoup de bien... ça me détend... mais il y a aussi un côté stimulant que j'aime... des fois ça m'arrive aussi de parler à des gens... quelques instants ... » (Extrait du cahier des observations, juin 2016). Les parcs possèdent en effet des propriétés à la fois stimulantes et calmantes qui procurent du bien-être aux participants.

Les espaces publics et les parcs tout particulièrement jouent un double rôle dans les trajectoires de rétablissement des jeunes gens. Ils agissent à la fois comme médiateurs lors des moments de crise et de souffrance et ils constituent également une source de stimulations agréables qui confère du bien-être aux participants dont les troubles sont stabilisés ou en rémission (Baumann et al., 2019; Codeluppi, 2016).

Chez de nombreux participants, la reconquête de l'espace urbain s'effectue dans un premier temps en groupe, puis de manière individuelle. Cela confère aux jeunes gens un sentiment de protection et de sécurité, comme l'explique Filipa, la responsable de l'équipe soignante : «[...] les jeunes... souvent quand ils vont mieux... ils partent explorer la ville en groupe... des fois ... pour certaines démarches ... administratives ou médicales... ils demandent à être accompagnés par l'un d'entre nous... ça les rassure ... » (Extrait d'entretien avec Filipa, août, 2016).

Dans la suite du travail, je détaille les ressources matérielles, sociales et sensorielles mobilisées au cours de la reconquête de l'espace urbain.

\subsubsection{Une mobilisation de ressources matérielles, sociales et sensorielles inédites}

Au fur et à mesure de leur reconquête de l'espace urbain, les participants mobilisent un nombre croissant et une diversité de ressources matérielles, sociales et sensorielles. Avec la rémission et la stabilisation des troubles, caractéristiques du cycle long, les participants exploitent ainsi davantage les ressources à disposition dans leur environnement (Duff, 2014 ; Codeluppi, 2016). Dans leur pratique de la ville, les jeunes gens activent ainsi les différentes «prises» de leur environnement et ils s'investissent davantage avec leur environnement (Golembiewski, 2013). Je dresse à ce propos une typologie des ressources matérielles, sociales et sensorielles utilisées par les participants dans leurs pratiques de reconquête urbaine.

Tout d'abord, les jeunes gens mobilisent des ressources matérielles inédites. Les participants se saisissent des objets à disposition dans leur environnement, ce qui leur permet de développer de nouvelles pratiques propices au bien-être. Les objets de loisirs comme des jeux de société ou encore des instruments de musique mis à disposition par l'institution sont fréquemment utilisés par les jeunes gens dans le but de médiatiser leur rapport à l'environnement. Ces objets leur permettent de développer de nouvelles pratiques qui leur procurent du bien-être, comme l'explique Jim, un jeune participant de vingt ans qui fréquente l'institution à mi-temps : «Depuis quelques temps... c'est vrai... j'aime bien jouer un peu de piano... celui qui se trouve dans la salle commune... j'en faisais encore il y a quelques années ... j'aime bien jouer des petits trucs... ça me change les idées... et puis des fois $\mathrm{j}$ ' ai quelques spectateurs (rires) qui viennent me demander ce que je joue... ou qui me demandent de jouer un truc pour eux ... du coup je vais chercher des partitions sur internet... et pis après je les amène à l'institution et je les joue ... c'est cool... j'aime bien » (Extrait du cahier des observations, juillet 2016). La musique constitue pour Jim une source de distraction, mais également un outil d'exploration et d'apprentissage qu'il mobilise au gré de ses envies et qui lui apporte du bien-être.

De même, les configurations spatiales de certains lieux font également partie des ressources matérielles mobilisées au cours du processus de reconquête spatiale. Même si, comme déjà mentionné plus haut, les espaces végétalisés sont encore souvent utilisés par les participants, ceux-ci privilégient désormais également d'autres lieux du centre-ville aux caractéristiques plus minérales. Ainsi, les places publiques mais également les rues piétonnes pourvues de petits aménagements tels que des bancs, des tables ou des marches d'escalier sont propices à une appropriation temporaire par les participants. Ces lieux constituent également d'excellents points d'observation de l'activité urbaine comme l'explique Dan, un jeune homme de vingt-et-un ans : «Quand je vais en ville... y a plein de coins que $\mathrm{j}$ 'aime bien... souvent je me pose... sur un banc dans la vieille-ville... ou alors sur les marches d'escalier vers la Riponne ou la cathédrale ... avec une bière ... et pis j'observe ce qui se passe autour de moi... de là-haut t'as une vue incroyable ... tu vois tout... tu te sens maître de la ville (rires)... au-dessus de tout le monde ... c'est vraiment cool» (Extrait du cahier des observations, juillet 2016). Ainsi, les espaces extérieurs avec un dégagement visuel à l'image de la cathédrale sont également appréciés par les participants, car ils leur confèrent un sentiment de contrôle sur leur environnement.

Les terrasses de café du centre-ville s'inscrivent également dans les ressources matérielles mobilisées par les participants. La configuration spatiale des terrasses offre un degré d'ouverture agréable sur la ville, tout en s'inscrivant dans un espace plus intime, celui des clients du café, comme l'explique Patrick : «Je me pose souvent sur les terrasses de café... c'est un peu comme un salon ouvert sur la ville... tu bois ton café tranquille... et pis tu peux observer ce qu'il se passe dans la rue... mais t'es pas dans 


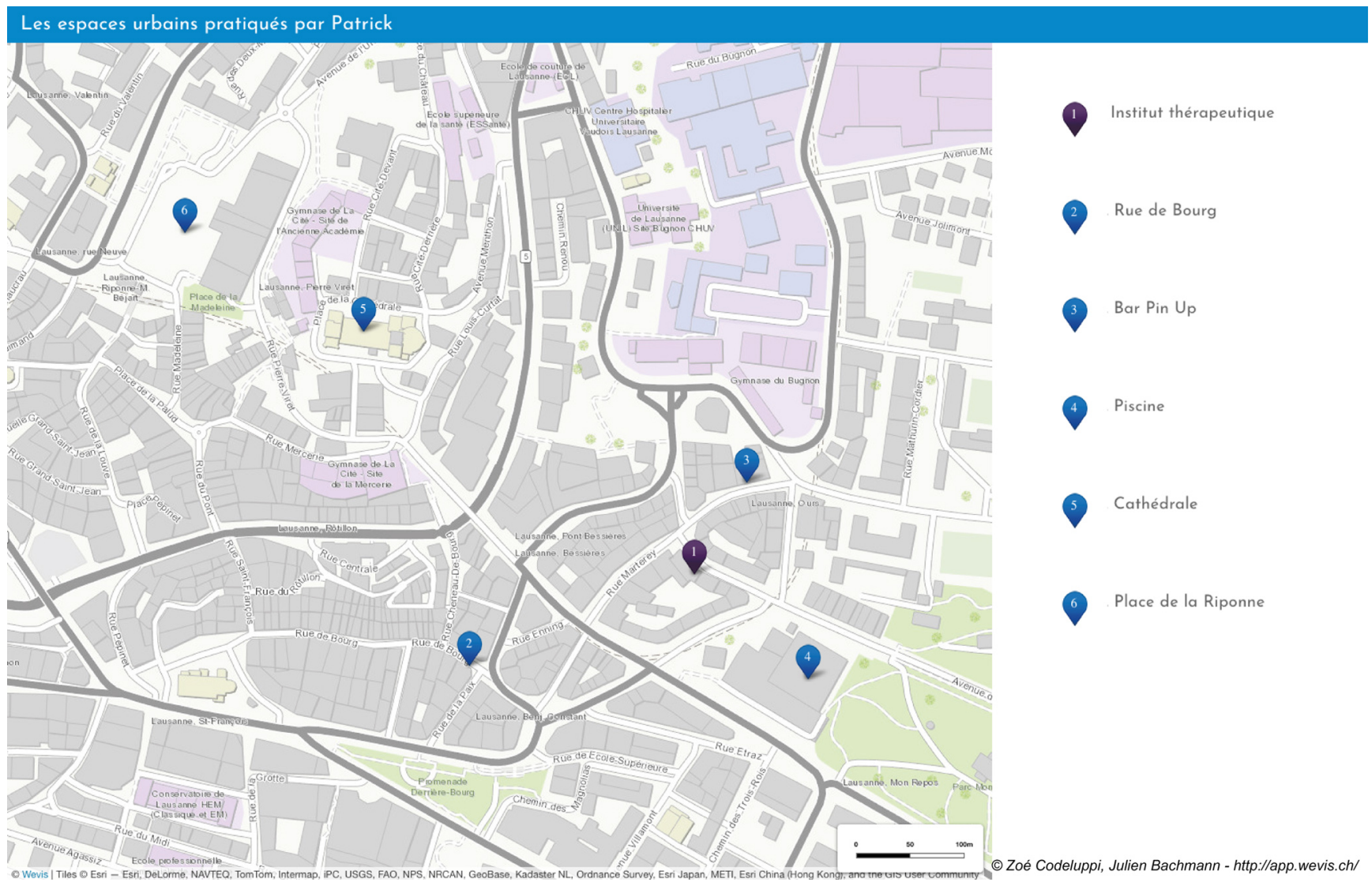

Figure 2. Les espaces de reconquête de Patrick.

l'action tu vois ... [...]» (Extrait du cahier des observations, juin 2016).

Enfin, les configurations spatiales de certains bâtiments publics, tel que le Palais Rumine qui abrite la bibliothèque cantonale et universitaire, sont considérées comme espaces de ressources. La présence de tables et de chaises dans divers coins du bâtiment, la gratuité d'accès aux expositions temporaires et à la bibliothèque, de même que l'esthétique de l'édifice confèrent du bien-être et du réconfort aux participants, comme l'explique Jim : «Quand je suis en ville... je fais un crochet par le Palais Rumine... souvent...j'aime bien m'arrêter devant et regarder le bâtiment... il est beau quand même... c'est artistique... les façades ... comme ça a été fait... après je me pose dans un des coins où il $\mathrm{y} a$ des tables et des chaises... j'écoute de la musique... je lis des livres ... des fois je vais à la bibliothèque ... je m'installe dans les fauteuils avec des journaux... je suis tranquille... Et pis quand j'ai envie je jette un oeil aux expos temporaires... des fois c'est intéressant... et pis c'est abrité quand il pleut j'aime bien venir là ... en plus c'est gratuit ...» (Extrait du cahier des observations, juillet 2016).

Ensuite, au cours de leur reconquête, les jeunes gens mobilisent des ressources sociales nouvelles. Les participants cherchent à établir des contacts avec des personnes fréquen- tant l'institution, mais également en dehors de celle-ci. Dans cette optique, les jeunes gens privilégient des espaces propices à l'interaction sociale ou à des activités en groupe qui permettent de nouer des contacts avec d'autres personnes. Les lieux tels que les cafés, les espaces publics ou la piscine municipale leur permettent de participer à l'activité sociale et de s'intégrer dans d'autres groupes de personnes.

À l'instar d'Elise, une jeune femme de vingt-et-un ans qui fréquente à mi-temps l'institution, les jeunes gens mobilisent souvent les jeux de société à l'institution pour établir de nouveaux liens avec d'autres membres. Ces jeux permettent en effet : «[...] d'apprendre à connaître les gens de l'institution ... et à se faire de nouveaux amis ... » (Extrait du cahier des observations, juillet, 2016). Les jeux de société constituent ainsi un médiateur qui permet à Elise d'élargir son réseau social au sein de l'institution.

D'autres participants comme Patrick cherchent davantage à établir un contact avec des personnes extérieures à l'institution. Patrick aime se rendre au café en raison de sa dimension sociale, comme il l'explique lui-même : «C'est pas rare qu'au café je parle avec des gens que je connais... et aussi que je connais pas... si tu es assis à une table à côté de quelqu'un qui est lui aussi tout seul... ben des fois ça arrive qu'on parle un moment de tout et de rien ... de banali- 
tés quoi... après quand c'est avec des gens que je connais pas... ben c'est facile... parce qu'ils connaissent pas ma situation... du coup c'est moins prise de tête... j'aime bien... après... quand des fois j'ai envie d'être seul... je prends le journal et pis j'évite les gens ... » (Extrait du cahier des observations, juillet 2016). Ainsi, le café offre à Patrick un espace social où il peut échanger avec ses connaissances mais aussi avec des inconnus. Ces interactions qui sont souvent éphémères confèrent à Patrick un certain anonymat qu'il apprécie. Il peut également réguler ses interactions, lorsqu'il en éprouve le besoin.

Dans une même perspective, Mike, un jeune homme de vingt ans, cherche lui aussi du contact avec des personnes extérieures à l'institution : «Mike se rend une fois par semaine à l'institution pour participer à l'activité piscine organisée par une soignante. Dans le cadre de cette activité, Mike a établi un lien avec une équipe d'étudiants universitaires venant s'entraîner aux plongeoirs tous les mercredis à la piscine. Par son statut de patient et grâce à une négociation avec les entraîneurs et les maîtres-nageurs, Mike a un accès aux plongeoirs qui d'habitude sont réservés aux groupes. Les entraîneurs et les étudiants l'ont intégré au groupe à tel point qu'ils le coachent lui aussi lors de ses plongeons, lui permettant d'acquérir une plus grande confiance en lui. Mike ne loupe jamais l'activité piscine, il aime beaucoup cette activité qui lui permet de s'intégrer à un autre groupe de jeunes grâce à ses performances physiques en plongeon qui lui confèrent une certaine reconnaissance sociale par la valorisation de ses capacités sportives » (Extrait du cahier des observations, juin 2016).

Cette activité permet à Mike de s'intégrer dans un groupe hors de l'institution thérapeutique et lui offre de nouvelles opportunités de rencontres, qui permettent de créer une relation affective bénéfique avec d'autres jeunes gens. Cette activité procure également un sentiment d'appartenance à Mike et un rôle dans lequel il se sent valorisé. Toutefois, les liens sociaux tissés avec des personnes extérieures à l'institution sont souvent éphémères et souples, de façon à pouvoir moduler l'interaction à la convenance des jeunes (Codeluppi, 2016).

Enfin, les participants privilégient des espaces urbains actifs et vivants dans lesquels ils peuvent puiser des ressources sensorielles agréables. Ainsi, les parcs publics mais également les terrasses de café ou encore les rues piétonnes sont appréciées par les jeunes gens pour les stimulations sensorielles que ces espaces offrent. Les sollicitations auditives comme le chant des oiseaux, le son du vent dans les arbres dans les parcs, mais également la rumeur de l'activité urbaine dans la zone piétonne du centre-ville, le bruit de l'eau des fontaines ou encore la musique douce des cafés sont autant de sollicitations qui confèrent aux participants un sentiment de bien-être (Codeluppi, 2016). Toutefois, à l'image de Patrick, le niveau de stimulations auditives, ne doit pas être trop intense. Un niveau de stimulation peu intense qui n'implique pas d'effort cognitif mais qui parvient à capter l'atten- tion, est vécu comme agréable par les participants (Baumann et al., 2019) ${ }^{12}$. De même, les stimulations olfactives et, en particulier, l'odeur de la forêt dans les parcs ou celle de la nourriture et du café dans les rues du centre-ville sont perçues agréablement par les jeunes gens et contribuent à leur confort. Ainsi, c'est un équilibre subtil entre ces différentes stimulations sensorielles de l'environnement qui procure du bien-être aux jeunes gens, participant ainsi à leur rétablissement.

\subsubsection{Une intensification des rapports à l'environnement}

Les observations montrent qu'au cours du cycle long, les participants s'engagent davantage, de façon plus active et plus intense avec une pluralité et une diversité de ressources présentes dans l'environnement (Thévenot, 2006 ; Corin, 2002). Cela se traduit par une participation plus active à la vie de l'institution et aux activités offertes mais aussi par la recherche de contact social et par la création de liens avec d'autres personnes dans et hors de l'institution. À l'image de Mike, Jim et Elise, les participants mobilisent un grand nombre et une grande diversité de ressources matérielles, sociales et sensorielles, pour la plupart inédites, qui témoignent ainsi d'un investissement plus intense des participants avec leur environnement. En effet, dans leur pratique de reconquête, les jeunes gens fréquentent de nouveaux espaces comme la piscine, la bibliothèque, les espaces publics ou encore les cafés. Ils mobilisent à cet effet une multitude de nouvelles ressources matérielles (mobilier urbain, éléments végétaux et minéraux de l'espace urbain), sociales (lieux de rencontres propices aux interactions sociales) et sensorielles (sollicitations auditives agréables).

L'engagement des jeunes gens avec les diverses «prises » de l'environnement requiert un effort d'ajustement et de coordination de leurs actions en fonction de celles des autres personnes (Thévenot, 2006). Cet ajustement est en effet indispensable, lorsque la portée de l'action est collective, c'està-dire qu'elle implique des interactions avec d'autres acteurs. Dans cette perspective, l'action se constitue à travers une coordination constante entre les diverses pratiques des acteurs impliqués dans celle-ci (Thévenot, 2006). Cette coordination requiert par ailleurs au préalable une compréhension des pratiques des autres acteurs, ainsi qu'un réapprentissage de la capacité à s'ajuster en fonction des pratiques d'autrui.

À l'exemple de Patrick, l'engagement avec l'environnement se traduit d'abord par une phase d'observation de la part des participants, dans le but de faciliter l'ajustement et la coordination avec les actions des autres. Cette phase d'observation est en effet indispensable pour les participants, puisqu'elle leur permet de mieux comprendre l'environnement, avant leur réapprentissage de la coordination, précé-

\footnotetext{
${ }^{12}$ L'étude de Baumann et al. (2019) emploie le terme de soft fascination pour désigner un niveau de stimulations agréable pour les participants et qui participe à leur rétablissement.
} 
dant la participation aux activités et aux situations quotidiennes : «À son arrivée à l'institution, Patrick est un jeune homme plutôt solitaire qui reste souvent en retrait. Il est présent aux activités mais n'y participe que rarement. Au fil des semaines, il s'ouvre peu à peu aux autres individus. Il prend davantage la parole en public, il discute avec les soignants et avec les autres jeunes de l'institution. Cette évolution est particulièrement notable dans le cadre de l'activité de danse. En effet, Patrick délaisse son rôle de spectateur au profit du rôle d'assistant de danse de la soignante en charge de l'activité. Peu à peu, le jeune homme s'investit dans l'activité. Il montre beaucoup de motivation et d'enthousiasme dans l'apprentissage des pas et des chorégraphies. Au cours des semaines, la soignante qui l'encourage beaucoup, le choisit comme partenaire de danse pour montrer les exercices et lui demande de l'assister dans l'animation de son activité. Un jour, peu avant le début de l'activité, Patrick me confie qu'il aime beaucoup cette activité qui lui procure de plaisir et l'aide beaucoup à se remettre de ses "problèmes" de santé. Il m'explique : "au début je me sentais un peu perdu... je connaissais pas vraiment la danse ... et puis j'avais un peu peur des autres ... mais maintenant ... ça m'a beaucoup aidé... surtout à être de nouveau avec d'autres ... à reprendre confiance en moi aussi" » (Extrait du cahier des observations, juin 2016). La participation de Patrick à l'atelier de danse témoigne de l'engagement progressif du jeune homme dans des activités dont la portée est collective. Après une phase d'observation qui lui permet de se réapproprier et de se familiariser avec les règles de l'activité de danse, le jeune homme réapprend progressivement à coordonner son action avec celle des autres. Patrick apprend en effet à reproduire les mouvements de l'animatrice et à coordonner ses pas avec les siens. Il interagit ensuite avec les autres participants pour corriger leur posture ou leurs pas. La pratique de la danse a une portée collective qui requiert donc un réapprentissage de la façon de coordonner son action en fonction de celle des autres (Thévenot, 2006).

Par ailleurs, l'engagement de Patrick dans les activités et les interactions, lui permet de nouer des contacts avec d'autres participants au cours des activités et facilite ainsi son intégration sociale. Grâce aux encouragements prodigués par l'animatrice et à son savoir-faire en tant que partenaire de danse, Patrick reprend confiance en lui et en ses capacités.

Dans cette même perspective, à l'image de Mike, d'autres participants réapprennent à se coordonner avec leur environnement mais hors de l'institution. Au cours de sa pratique du plongeon dans le cadre de l'activité piscine décrite précédemment, Mike cherche en effet à coordonner son action en fonction de celle des étudiants, à travers la reproduction des figures proposées par les entraîneurs du groupe. La participation de Mike aux activités du groupe d'étudiants, témoigne de son investissement dans des interactions sociales avec des personnes hors de l'institution. Le jeune homme participe activement aux conversations avec les étudiants et les entrâneurs qui l'encouragent dans l'exécution de ses plongeons et qui lui montrent comment réaliser ces figures. À l'exemple de Patrick et de Mike, les participants s'approprient ainsi de nouvelles ressources sociales qui leur procurent du bien-être et participent à leur rétablissement.

\section{L'espace public urbain comme ressource thérapeutique}

Ainsi, comme l'a montré ce travail, le milieu urbain joue actuellement un rôle incontestable dans le rétablissement des jeunes personnes schizophrènes (Söderström et al., 2016, 2017). Pour comprendre la place occupée par l'espace urbain dans les soins psychiatriques, il faut remonter à l'ère de la désinstitutionalisation qui a contribué à la décentralisation des espaces de soins et à l'implantation de services communautaires en ville, au plus près de la société, afin d'encourager une progressive intégration des ex-patients du système asilaire au sein de la collectivité. Aujourd'hui, la prise en charge et le suivi des personnes souffrant de troubles psychiques sont plus personnalisés et s'ancrent dans les lieux de vie de celles-ci. À cet effet, des équipes de soins mobiles et des partenariats entre différents acteurs du réseau de soins sont créés, afin d'accompagner les individus dans leur vie quotidienne (Chevalley, 2016). Même si les espaces institutionnels demeurent mobilisés à certains moments du rétablissement, celui-ci s'accomplit essentiellement hors des murs de l'institution.

Bien que la vie en milieu urbain puisse à certains moments du rétablissement se révéler problématique pour les personnes schizophrènes, car source de stress et d'inconfort, mon analyse montre qu'à l'inverse, l'espace urbain offre également une multitude de ressources matérielles sociales et sensorielles mobilisables selon les besoins.

Mon travail avait comme objectif principal d'analyser l'évolution de la spatialité en matière de pratiques et de ressources de rétablissement des jeunes personnes souffrant de schizophrénie, en fonction de la temporalité non-linéaire, caractéristique de ces troubles psychiques. Dans cette optique, mon travail se base sur un terrain ethnographique de trois mois dans une institution thérapeutique lausannoise avec de jeunes participants ayant vécu un premier épisode psychotique datant de moins de trois ans.

Mes résultats mettent en évidence quatre aspects centraux. Premièrement, la temporalité du rétablissement de la schizophrénie se caractérise par une logique non-linéaire et cyclique. Elle fait intervenir le cycle long qui correspond au processus long du rétablissement, depuis le vécu d'un épisode à la progressive stabilisation et rémission des troubles et le cycle court, c'est-à-dire les moments temporaires d'expression des troubles qui interviennent tout au cours du cycle long.

Deuxièmement, mon analyse met en exergue l'extension du périmètre des pratiques des jeunes gens depuis l'échelle du quartier de l'institution à l'échelle de la ville entière. À cet 
effet, je montre l'évolution des pratiques de retrait des jeunes gens, caractéristiques du cycle court vers des pratiques de reconquête de l'espace urbain, propres au cycle long.

Troisièmement, mon étude montre que les participants mobilisent une pluralité et une diversité de ressources matérielles, sociales et sensorielles de leur environnement, au fur et à mesure de leur reconquête de l'espace urbain. Je propose à cet effet une typologie qui distingue les ressources familières, utilisées lors des périodes de manifestation des troubles, des ressources novatrices dont se saisissent les jeunes gens au fur et à mesure de l'amélioration de leur état psychique.

Enfin, mon travail emploie le concept «d'intensité d'engagement » développé par Thévenot (2006) pour rendre compte avec précision du degré d'investissement des participants dans les relations avec les ressources matérielles, sociales et sensorielles de l'environnement. L'analyse montre qu'au fur et à mesure du rétablissement, les jeunes gens s'engagent avec une plus grande quantité et avec une plus grande diversité de ressources.

De manière générale, une meilleure compréhension des spatialités et des temporalités complexes de rétablissement des personnes schizophrènes permet de contribuer aux réflexions très actuelles des professionnels de la santé mentale qui portent sur l'implémentation de nouvelles stratégies thérapeutiques en milieu urbain et à Lausanne tout particulièrement (Baumann et al., 2019).

Au-delà de la pratique des espaces institutionnels de soins implantés en milieu urbain, mon analyse fait ressortir la place prépondérante occupée par l'espace public urbain dans les trajectoires de rétablissement des jeunes participants, ainsi que leur dimension complémentaire. Quand bien même l'étude montre le rôle de refuge joué par les différents espaces institutionnels de soins, comme l'hôpital psychiatrique, les consultations ambulatoires ou encore les hôpitaux de jour, véritables lieux-clés dans les mécanismes de rétablissement des jeunes participants, le travail souligne également la façon dont ces derniers s'approprient une variété d'espaces publics, aux ressources matérielles sociales et sensorielles très diverses. Les parcs et les édifices publics comme les bibliothèques ou les églises jouent tout particulièrement un rôle important dans les moments de crises (Baumann et al., 2019). La présence de végétation, la présence de mobilier urbain invitant à la pause, mais également l'anonymat et le calme sont autant de ressources offertes par ces espaces et mobilisées par les participants dans les périodes de grande souffrance. De même, les parcs, les rues piétonnes, les cafés ou les places publiques sont des espaces appropriés par les participants au cours de la rémission des troubles. Les lieux de rencontres, la présence d'éléments minéraux, mais aussi les opportunités d'interactions et d'inclusion sociale dans le cadre d'échanges ou d'activités de groupes, mais également les stimulations sensorielles comme les rumeurs de la ville ou la musique constituent les ressources privilégiées des participants en cours de rémission.
Dans cette perspective, il semble opportun d'intégrer les espaces publics urbains aux stratégies thérapeutiques des professionnels de la santé mentale, tant dans les moments de souffrance qu'au cours de la période de rémission des troubles et de réfléchir à leur complémentarité avec les diverses institutions de soins, qui elles aussi jouent un rôle clé tout au long du rétablissement.

Des activités thérapeutiques individuelles ou collectives organisées dans des espaces verts ou dans des lieux publics comme des musées, par exemple, permettraient d'encourager les patients à s'approprier des lieux propices au bien-être et mobilisables en cas de crise. Une stratégie similaire pourrait être développée dans des espaces actifs et «pro-sociaux » offrant un potentiel d'interactions avec d'autres et d'inclusion sociale, comme les places publiques, la piscine, les musées ou la patinoire (Okkels et al., 2018 ; Baumann et al., 2019).

D'autre part, le développement d'espaces de consultation thérapeutiques plus flexibles et modulables, en proposant par exemple, des lieux de rencontres dans d'autres espaces comme des cafés, participerait à proposer une large gamme de lieux qui pourraient être adaptés aux besoins des patients, selon leur état de santé psychique (Baumann et al., 2019; Codeluppi, 2016).

De même, l'accompagnement par un professionnel de la santé mentale de confiance dans le cadre de différentes activités ou d'obligations quotidiennes en ville, permettrait d'assister les patients dans leurs pratiques de mobilité en ville et de développer les connaissances géographiques, propices à leur autonomisation spatiale (Baumann et al., 2019).

En outre, des stratégies thérapeutiques alliant marche urbaine et discussions, effectuées de façon collective ou individuelle, participerait à la création d'un nouvel espace de thérapie mobile, au cours duquel les patients seraient amenés à développer leur mobilité au sein de la ville, tout en s'engageant dans une interaction avec d'autres interlocuteurs (Baumann et al., 2019).

Enfin, si les espaces publics urbains participent activement au processus de rétablissement des jeunes schizophrènes, l'analyse souligne aussi le rôle des objets technologiques embarqués, comme les ordinateurs, les tablettes ou encore les téléphones portables qui médiatisent le rapport des personnes à leur environnement, réduisant ainsi le sentiment de stress et d'anxiété. À ce propos, le potentiel de ces supports dans le développement de démarches et de stratégies thérapeutiques destinées aux personnes souffrant de troubles psychiques mériterait d'être approfondi dans des travaux ultérieurs.

Disponibilité des données. L'ensemble des données utilisées dans le cadre de ce travail (entretiens, observations) n'a pas été rendu public, ni accessible en raison du caractère sensible et confidentiel du matériel récolté qui a trait à l'état de santé psychique, au traitement et au rétablissement des participants. Pour des raisons de sécurité et de protection de la sphère privée des participants et des 
soignants, l'accès à ces données est strictement réservé à l'auteur ainsi qu'à l'équipe de recherche dont l'auteur fait partie. Les chercheurs sont par ailleurs tous soumis aux règles de confidentialité usuelles concernant les données sensibles. L'ensemble des données est stocké sur un serveur sécurisé de l'Université de Neuchâtel.

Intérêts concurrents. L'auteur déclare qu'elle n'a aucun conflit d'intérêt.

Remerciements. Je tiens à remercier les patients et les soignants de l'institution thérapeutique lausannoise pour leur disponibilité et leur collaboration tout au long de ma recherche.

Edited by : Myriam Houssay-Holzschuch

Reviewed by : two anonymous referees

\section{Références}

Baumann, P. S., Empson, L. A., Söderström, D., Codeluppi, Z., Golay, P., Söderström, O., and Conus, P. : Urban remediation : a new recovery oriented strategy to manage city stress after first episode psychosis, 2019.

Bister, M., Klausner, M., and Niewöhner, J. : The cosmopolitics of "niching", Rendering the city habitable along infrastructures of mental health care. Urban Cosmopolitics. Agencements, assemblies, atmospheres, London, Routeledge, 2016.

Chevalley, C. : Paysage de la santé mentale lausannois : état des lieux de ses espaces et de ses visions de soins de 1960 à nos jours, Mémoire de master, Neuchâtel, Université de Neuchâtel, https: //doc.rero.ch/record/277776/files/Cbevalley_C_line_Paysage_ de_la_sant_mentale_lausannois_UNINE_MEMOIRE_2016.pdf (la date du dernier accès : 12 novembre 2018) 2016.

Codeluppi, Z. : Entre le plein et le vide : les espaces-temps quotidiens de jeunes patients souffrant de troubles psychotiques en milieu urbain, Géo-Regards, 9, 119-133, 2016.

Corin, E. : Se rétablir après une crise psychotique : ouvrir une voie ? Retrouver sa voix ?, Santé mentale au Québec, 27, 65-82, 2002.

Corin, E. : À l'ombre de la psychose ... des tracés en creux aux marges de la culture, Cahiers de psychologie clinique, 2, 197218, 2003

Curtis, S. : Space, Place and Mental Health, London, Routledge, 316 pp., 2010.

Davidson, L. : Living Outside Mental Illness, Qualitative Studies of Recovery in Schizophrenia, New York, NYU Press, 227 pp., 2003.

De Vries, S., Verheij, R. A., Groenewegen, P. P., and Spreeuwenberg, P. : Natural Environments - Healthy Environments ? An Exploratory Analysis of the Relationship between Greenspace and Health, Environ. Plann. A, 35, 1717-1731, https://doi.org/10.1068/a35111, 2003.

Duff, C. : Exploring the role of "enabling places" in promoting recovery from mental illness : A qualitative test of a relational model, Health Place, 18, 1388-1395, 2012.

Duff, C. : Assemblages of Health : Deleuze's Empiricism and the Ethology of Life, Springer, Dortrecht, 209 pp., 2014.
Duff, C. : Atmospheres of recovery : Assemblages of health, Environ. Plann. A, 48, 58-74, https://doi.org/10.1177/0308518X15603222, 2015.

Evered, E. : The role of the urban landscape in restoring mental health in Sheffield, UK : service user perspectives, Landscape Res., 41, 678-694, 2016.

Estroff, S. E. : Making it crazy : An ethnography of psychiatric clients in an American community, University of California Press, London, 1985.

Faris, R. E. L. and Dunham, H. W. : Mental disorders in urban areas : an ecological study of schizophrenia and other psychoses, Oxford, Univ. Chicago Press, England, 270, 1939.

Gleeson, B. : Geographies of Disability, Routledge, London/New York, 243 pp., 1999.

Golembiewski, J. : Lost in space : The place of the architectural milieu in the aetiology and treatment of schizophrenia, Facilities, 31, 427-448, 2013.

Hennink, M. M. : Focus group discussions : Understanding Qualitative Research, Oxford University Press, 213 pp., 2014.

Kapur, S. : Psychosis as a state of aberrant salience : a framework linking biology, phenomenology and pharmacology in schizophrenia, Am. J. Psychiat., 160, 13-23, https://doi.org/10.1176/appi.ajp.160.1.13, 2003.

Kelly, B. D., O'Callaghan, E., Waddington, J. L., Feeney, L., Browne, S., Scully, P. J., Clarke, M., Quinn, J. F, Mc Tigue, O., Morgan, M. G., Kinsella, A., and Larkin, C. : Schizophrenia and the city : A review of the literature and prospective study of psychosis and urbanicity in Ireland, Schizophr. Res., 116, 75-89, https://doi.org/10.1016/j.schres.2009.10.015, 2010.

Kirkbride, J. B., Fearon, P., Morgan, C., Dazzan, P., Morgan, K., Murray, R. M., and Jones, P. B. : Neighbourhood variation in the incidence of psychotic disorders in SE London, Psychiatry Epidemiology, 42, 438-445, https://doi.org/10.1007/s00127007-0193-0, 2007.

Knowles, C. : Burger King, Dukin Donuts and community mental health care, Health Place, 6, 213-224, 2000.

Klausner, M. : Choreografien psychiatrischer Praxis : eine ethnografische Studie zum Alltag in der Psychiatrie, transcript Verlag, Bielefeld, 2014.

Lapassade, G. : La méthode ethnographique, DESS d'Ethnométhodologie et Information, année scolaire, 1992-1993, Université Paris 8 Vincennes-St-Denis, accessible à l'adresse suivante : http://vadeker.net/corpus/lapassade/ethngrso.htm (la date du dernier accès : le 14 novembre 2018), 1993.

Lefèbvre, H. : La production de l'espace, L'Homme et la société, 31, 15-32, 1974.

Lysaker, P. and Lysaker, J. : Schizophrenia and the fate of the self, University Press Oxford, Oxford, 2008.

McGrath, L. and Reavey, P. : Zip me up, and cool me down : Molar narratives and molecular intensities in "helicopter" mental health services, Health Place, 38, 61-69, 2016.

Milligan, C. and Wiles, J. : Landscapes of care, Progress in Human Geography, 34, 736-754, 2010.

Myin-Germeys, I., Oorschot, M., Collip, D., Lataster, J., Delespaul, P., and van Os, J. : Experience sampling research in psychopathology : opening the black box of daily life, Psychol. Med., 39, 1533-1547, 2009.

Okkels, N., Kristiansen, C. B, Munk-Jorgensen, P., and Sartorius, N. : Urban mental health : challenges and 
perspectives, Curr. Opin. Psychiatr., 31, 258-264, https://doi.org/10.1097/YCO.0000000000000413, 2018.

Parr, H. : Delusional Geographies : The Experiential Worlds of People during Madness/Illness, Environ. Plann. D, 17, 673-690, https://doi.org/10.1068/d170673, 1999.

Parr, H. : Mental health, the arts and belongings, T. Brit. Geogr., 31, 150-166, 2006.

Parr, H. : Mental Health and Social Space : Towards Inclusionary Geographies ?, Blackwell publishing Ltd, Oxford, UK, 2008.

Philo, C. and Wolch, J. : The "three waves" of research in mental health geography : a review and critical commentary, Epidemiol. Psichiat. S., 10, 230-244, 2001.

Poirel, M.-L., Khoury, E., Rodriguez, L., Larivière, N., and Riendeau-Janvier, C. : Contribution des hôpitaux de jour en psychiatrie dans le champ contemporain de la santé mentale au Québec, Ciência \& Saúde Coletiva, 18, 2929-2937, 2013.

Söderström, O., Empson, L. A, Codeluppi, Z., Söderström, D., Baumann, P. S., and Conus, P. : Unpacking "the City" : an experienced based approach to the role of urban living in psychosis, Health and Place, 42, 104-110, https://doi.org/10.1016/j.healthplace.2016.09.002, 2016.

Söderström, O., Söderström, D., Codeluppi, Z., Empson, L. A., and Conus, P. : Emplacing recovery : how persons diagnosed with psychosis handle stress in cities, Psychosis, 9, 1-8, https://doi.org/10.1080/17522439.2017.1344296, 2017.
Thévenot, L. : L'action au pluriel : sociologie des régimes d'engagement, Éd. La Découverte, 111, 2006.

van Os, J. : Does the urban environment cause psychosis ?, Br. J. Psychiatry, 184, 287-288, 2004.

van Os J., Rutten, B. P., and Poulton, R. : Gene-environment interactions in schizophrenia : review of epidemiological findings and future directions, Schizophrenia Bull., 34, 1066-1082, https://doi.org/10.1093/schbul/sbn117, 2008.

Vassos, E., Pedersen, C. B., Murray, R. M., Collier, D. A, and Lewis, M. C. : Meta-analysis of the association of urbanicity with schizophrenia, Schizophrenia Bull., 38, 1118-1123, https://doi.org/10.1093/schbul/sbs096. 2012.

Verdoux, H. and Cougnard, A. : The early detection and treatment controversy in schizophrenia research, Curr. Opin. Psychiatr., 16, 175-179, https://doi.org/10.1097/00001504-200303000-00006, 2003.

Yates, I., Holmes, G., and Priest, H. : Recovery, place and community mental health services, J. Ment. Health, 21, 104-113, 2012. 